\title{
Breast-feeding and brain development
}

\author{
Kim Fleischer Michaelsen', Lotte Lauritzen', \\ Marianne Hørby Jørgensen ${ }^{2}$ and Erik Lykke Mortensen ${ }^{3,4}$
}

'Department of Human Nutrition, The Royal Veterinary and Agricultural University, Frederiksberg; ${ }^{2}$ Paediatric Department, Hillerød Hospital; ${ }^{3}$ Danish Epidemiology Science Center, Institute of Preventive Medicine, Copenhagen University Hospital, Copenhagen; ${ }^{4}$ Department of Health Psychology, Institute of Public Health, University of Copenhagen, Copenhagen, Denmark

Abstract

The effect of breast-feeding on brain development, especially cognitive and visual, has been examined in many studies. The aim of this article is to summarize this group's studies and relate them to results from reviews and meta-analyses. In two separate studies of visual development in term infants, visual acuity was better in breast-fed than in formula-fed infants, which is in accordance with the results from a meta-analysis. Among studies examining the association between breast-feeding and cognitive development most find a positive association, even when controlling for relevant confounding factors. A positive effect was found in two different samples of young adults using two different intelligence quotient tests. The most plausible explanation for this effect is the differences in composition between human milk and infant formula (e.g. docosahexaenoic acid). It could also be because mothers who choose to breast-feed on average stimulate and support their infants better. Further, unidentified factors may also correlate with both infant feeding and development of cognitive and intellectual ability, so-called residual confounding.

Keywords: breast-feeding; cognitive ability; docosahexaenoic acid; infant feeding; intelligence quotient (IQ); LCPUFA; mental development; visual acuity; visual evoked potentials

\section{Introduction}

The effect of breast-feeding on brain development has been examined in many studies, especially two aspects of brain development, cognitive development and the development of visual acuity. If there is a positive effect of breast-feeding, several potential mechanisms could explain the effect. One of the most plausible explanations is that the effect is caused by the breast-milk content of long-chain polyunsaturated fatty acids (LCPUFA), especially the $n-3$ fatty acid docosahexaenoic acid (DHA), which accumulate in neural membranes during infancy (1). The aim of this article is to give a short summary of the present authors' studies within the field and relate them to results from reviews and meta-analyses.

Breast milk contains environmental contaminants such as methylmercury and polychlorinated biphenyls (PCBs) at higher levels than infant formula. Prenatal exposures to high levels of these substances have been shown to have negative effects on brain development $(2,3)$, and theoretically the additional exposure of these contaminants through breast-feeding could have a negative effect on development, even though the prenatal brain seems much more vulnerable (4). A recent study suggested that PCBs transmitted through breast milk could have a negative effect on neurodevelopment (5). However, the data are not conclusive, since it is difficult to differentiate between prenatal and postnatal exposure (4).

Studies that compare breast-fed with non-breastfed infants are, by definition, observational since it is unethical to randomize infants to breast-feeding. In most industrialized countries breast-feeding is more prevalent among mothers with both high education and high social status, and most studies find a positive association between intelligence and education and between intelligence and socioeconomic status. Consequently, it is essential that studies comparing indicators of brain development in breast-fed and non-breast-fed infants control for potential confounders such as maternal education, family socioeconomic status and, if possible, maternal intelligence. 


\section{Visual development}

During infancy visual acuity gradually increases until it reaches a plateau around the age of 12 months (6). Visual acuity has been assessed mainly by two different methods: behaviourally and electrophysiologically based methods. The main behavioural method, the Teller acuity card procedure or "forced choice preferential looking", evaluates an infant's tendency to gaze at a pattern, rather than a blank field. The infant is presented with gratings of different spatial frequencies and the observer looks at the infant and decides which gratings attract the gaze of the infant. The procedure is somewhat subjective and requires an integrated behavioural response that includes factors other than strictly visual ones (e.g. alertness, attention and motor control). In the electrophysiologically based methods one measures visual evoked potentials (VEPs) in the visual cortex, the amplitude of which decreases with increasing spatial frequencies, and the threshold gives the visual acuity. These methods are far less subjective and specifically based on visual factors, although they also involve the process of signal conduction from the eye to the visual cortex.

In an observational study of two groups of healthy term infants, either being breast-fed or receiving infant formula, visual acuity was examined with Teller cards at the age of 1,2 and 4 months (7). Visual acuity increased significantly from 1 to 4 months in both groups, but the increase was significantly higher in the breast-fed group (Fig. 1). This was paralleled by a decrease in DHA of red blood cells in formula-fed infants and with a significantly lower level at 2 and 4 months compared with breast-fed infants.

A randomized intervention study tested the effect of supplementing infant formula with DHA on visual acuity by swept visual evoked potentials (SWEEP-VEP) in healthy term infants (8). A control group of breast-fed infants was included for comparison. The formulae were given from the age of 1-4 months and visual acuity was measured at the age of 4 months. Breast-fed infants had significantly better visual acuity than infants receiving a standard infant formula $(p=0.02)$. The visual acuity of infants receiving a formula supplemented with DHA had a mean visual acuity between these two groups, but not significantly different from the group receiving standard infant formula.

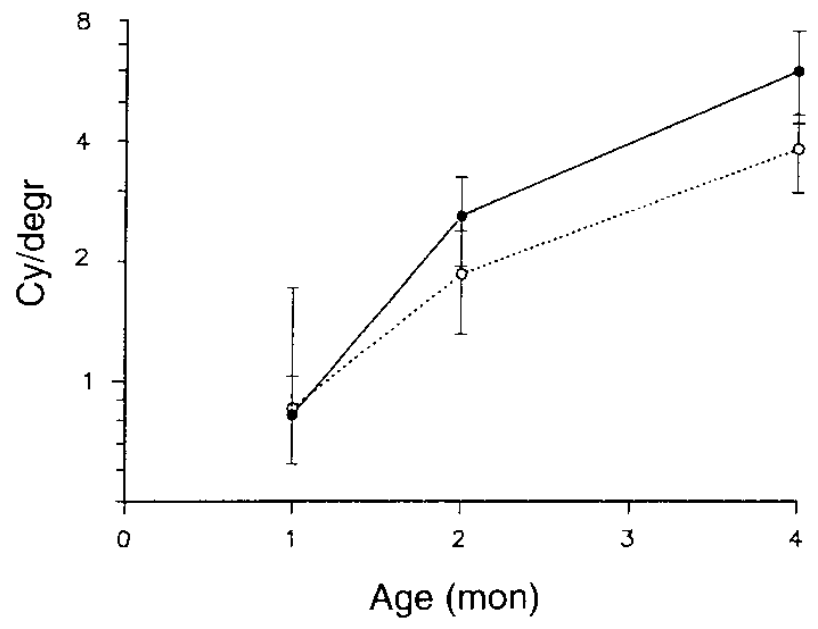

Fig. 1. Visual acuity examined by Teller cards in healthy term breast-fed and formula-fed infants. Bars are 95\% confidence intervals. O: Breast-fed infants $(n=17)$; $\bigcirc$ : formula-fed infants $(n=16)$; Cy/degr: cycles per degree. The increase in visual acuity was significantly higher in breast-fed infants $(p<0.001)$. (Reproduced from Ref. (7) with kind permission from Lipids.)

Another observational study examined visual acuity by SWEEP-VEP in a group of 39 term breast-fed 4-month-old infants and related it to the DHA content of the mothers' milk (9). Forty-seven per cent of the variation in milk DHA content could be explained by maternal fish intake. In this crosssectional study there was a significant positive association $(p=0.02)$ with higher visual acuity the more DHA the mother had in her milk.

The studies examining the effects of dietary LCPUFA on visual acuity in infants were analysed in a systematic review by SanGiovanni et al. (10). They divided the studies into those using behaviourally based methods such as Teller acuity cards and those using electrophysiologically based methods such as SWEEP-VEP. Furthermore, they divided them into randomized studies testing two kinds of infant formula and non-randomized studies comparing breast-fed and formula-fed infants. Among the non-randomized studies they found that breastfed infants had significantly better visual acuity at the age of 2 months $(0.49$ octaves, $p<0.000001)$ and 4 months $(0.18$ octaves, $p=0.04)$ and using electrophysiological methods breast-fed infants had a significantly better acuity at 4 months $(0.37$ octaves, $p=0.02)$. The conclusion, which also covered the randomized studies with formula supplemented with $n-3$ fatty acids, was that some aspects of dietary $n-3$ fatty acid intake are associated with performance on visual acuity tasks at 2 and, possibly, 4 months of age in healthy term 
infants, while it was still open to question whether $n-3$ intake confers a lasting advantage in the development of visually based processes.

\section{Cognitive development}

Two meta-analyses, published in 1999 and 2000, examined the association between breast-feeding and cognitive development. Anderson et al. (11) identified 20 studies that met three inclusion criteria: (i) comparing predominantly breast-fed with predominantly formula-fed; (ii) using a widely applied test of cognitive development yielding a single score; and (iii) testing subjects between infancy and adolescence. The included studies assessed cognitive development at ages between 6 months and 15 years. Eleven of the studies controlled for at least five covariates and presented both unadjusted and adjusted results. Six of the studies included a measure of maternal intelligence quotient (IQ) in the analysis. The unadjusted benefit of breast-feeding was 5.3 IQ points, but after adjustment for appropriate covariates the increment was still 3.16 and highly significant $(95 \%$ confidence interval 2.35-3.98) (Fig. 2). In addition, the effect was supported by a significant dose-response

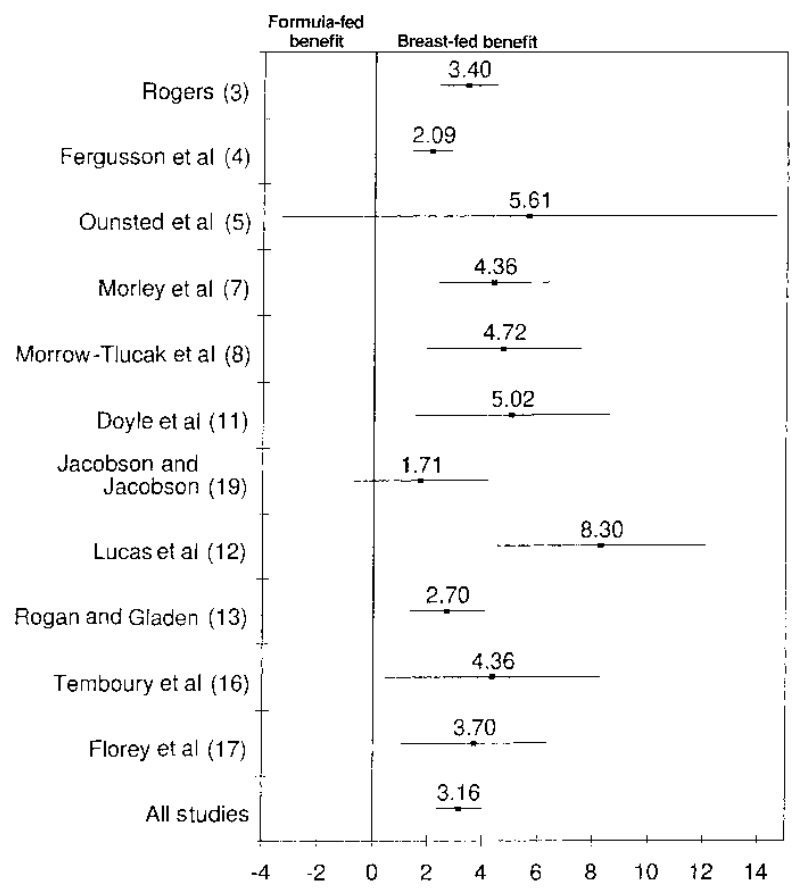

Fig. 2. Meta-analysis of 11 studies which controlled for at least five covariates and presented adjusted intelligence quotient scores for the mean difference between formula-fed and breast-fed infants. NB. The reference numbers in the figure do not relate to those in the present article. (Reproduced from Ref. (11) with kind permission from Am J Clin Nutr.) effect, with better cognitive development with increased duration of breast-feeding. For the following categories of duration of breast-feeding, 4$7,8-11,12-19,20-27$ and $\geq 28$ weeks, the effects of breast-feeding were $-0.02,1.68,2.15,2.78$ and 2.91 IQ points, respectively. All of the estimated effects, except for the 4-7 week category, were significant $(p<0.001)$. In an analysis by category of birth weight they found that the effect of breastfeeding was significantly larger in children with low birth weight (5.18 IQ points) than in those with normal birth weight (2.66 IQ points). This finding supports the LCPUFA hypothesis, as preterm infants are born with a low LCPUFA status and thereby more dependent on LCPUFA supply (1). Another finding from the analysis was that the effect seemed to be independent of the age at which the outcome was examined. Thus, the effect seen early in life seems to be stable throughout childhood.

In the review by Drane and Logemann 24 studies were evaluated (12). There is considerable overlap between the studies included in this review and the studies included in the meta-analysis of Anderson et al. (11), but several studies were only included in one of the two reviews. Drane and Logemann did not perform a meta-analysis but concluded that four out of six studies fulfilling strict inclusion criteria found a 2-5 IQ point advantage of breastfeeding for term infants and an 8 point advantage for low-birth-weight infants. They concluded that "the question of whether breast-feeding and formula feeding have differential effects on cognitive development has not yet been comprehensively answered". In an interesting discussion they tried to calculate the potential impact of type of infant feeding on the prevalence of developmental disability in the US population. Assuming a normal distribution of IQ in a population and a mean of 95 , a 5 point upward shift in the population to a mean of 100 will result in a decrease in the percentage of children with an IQ $<85$ from $26 \%$ to $16 \%$. Using these figures they estimated that the cost of special education in the USA could be reduced from $\$ 4.5$ billion to $\$ 3.9$ billion if the percentage of children who were predominantly breast-fed at 4 months was increased from $20 \%$ to $50 \%$.

The effects of breast-feeding on IQ were examined in the Copenhagen Perinatal Cohort Study (13). The cohort comprises 9125 infants born from 
1959 to 1961. Demographic, socioeconomic, prenatal and postnatal medical data were recorded. Information about duration of breast-feeding was collected at an interview with the mother when the infant was 12 months old. In the analysis total duration of breast-feeding (exclusive or partial) was used. Duration of breast-feeding was divided into five groups: $\leq 1$ month, $2-3$ months, $4-6$ months, 7-9 months and $>9$ months. Intelligence was assessed as adults in two non-overlapping subgroups. A group of 973 men and women was examined with the Wechsler Adult Intelligence Scale (WAIS) at a mean age of 27.2 years. For male participants in the cohort, draft records were obtained from the military authorities. These include a $45 \mathrm{~min}$ intelligence test (Børge Priens Prøve), with a score range from 0 to 78 . Complete data were available for 2280 men with a mean age of 18.7 years. Thirteen potential confounders were included as covariates in the analysis: parental social status and education, single mother status, mother's age, height and weight gain during pregnancy, cigarette consumption during the third trimester, number of pregnancies, estimated gestational age, birth weight, birth length, indexes of pregnancy and delivery complications. In both samples a highly significant dose-response relationship was found, indicating a higher IQ with a longer duration of breast-feeding (Fig. 3). In both samples there were no significant differences between the categories of 7-9 months and >9 months' breastfeeding, but apparently breast-feeding beyond 9 months was not associated with additional IQ benefits. The WAIS full-scale IQ increased from 99.4 in those breast-fed for $\leq 1$ month to 106.0 in those breast-fed for 6-9 months. This difference corresponds to about half an SD. For the conscript sample the scores increased from 38.0 in the group breast-fed for $\leq 1$ month to 40.1 in the group breast-fed for 7-9 months. The difference between these two groups is less that one-fifth of an SD. Full-scale WAIS IQ $<90$ can be regarded as suboptimal performance. The percentage of subjects with values $<90$ was significantly higher for the shorter duration of breast-feeding, with $28 \%$ in those breast-fed for $\leq 1$ month and only $4 \%$ in those breast-fed $>9$ months (Fig. 4).

\section{Conclusion}

Several studies, including some of the authors' own results, have shown better visual acuity in breast-fed
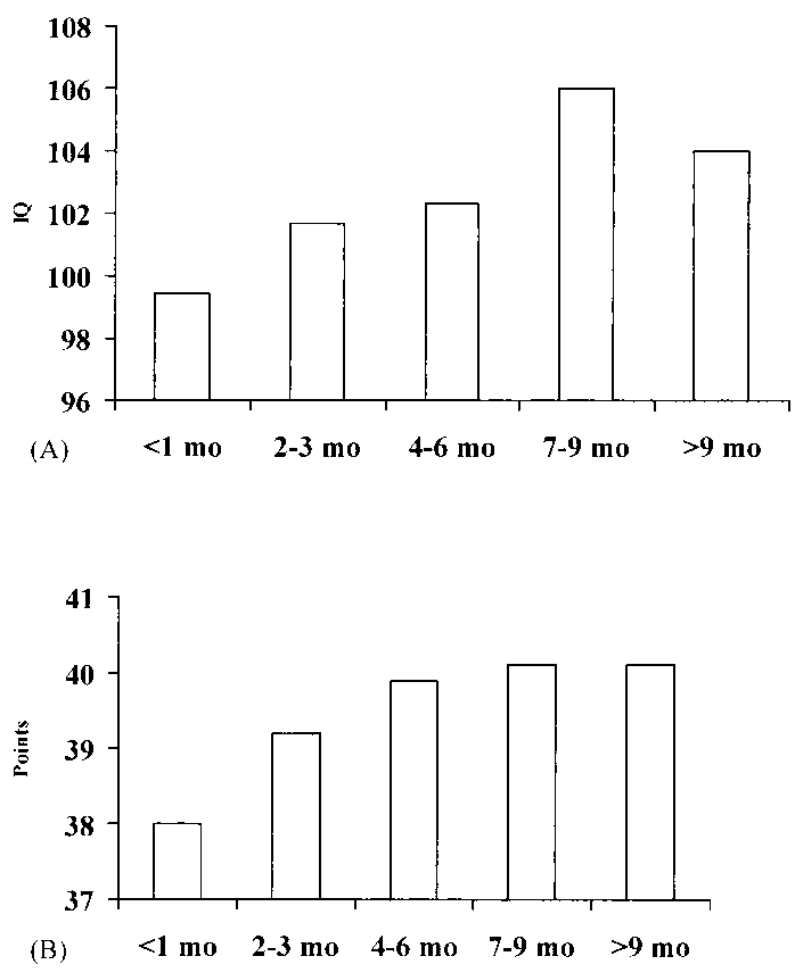

Fig. 3. Adjusted means for intelligence test scores in relation to duration of breast-feeding from the Copenhagen Perinatal Cohort (13). (A) Wechsler Adult Intelligence Scale (WAIS) full-scale intelligence quotient (IQ) for 973 men and women with a mean age of 27.2 years. (B) Data from 2280 male conscripts tested at a mean age of 18.7 years with the Børge Priens Prøve test. The difference between the feeding groups was significant in both samples: (A) $p<0.001$, (B) $p=0.01$.

than in formula-fed infants. Intervention studies with infant formula have suggested that such a difference could be explained by differences in $n-3$ fatty acid intake. However, since the studies comparing breast-fed and non-breast-fed infants are not randomized, other factors or residual confounding cannot be ignored. It is not known whether these differences in visual acuity early in life have any long-term effects.

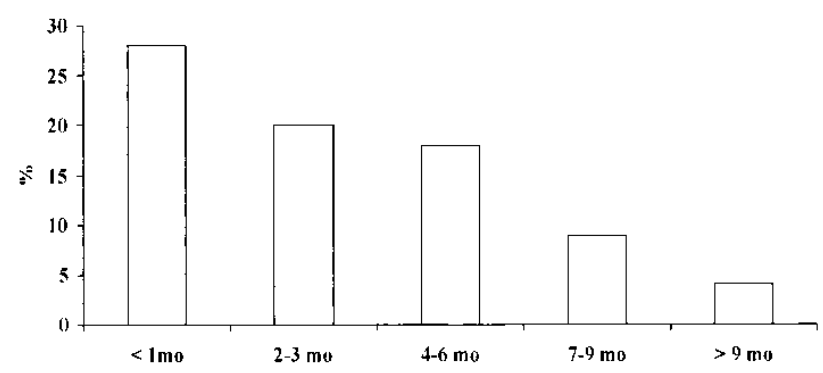

Fig. 4. Percentage of subjects with full-scale Wechsler Adult Intelligence Scale (WAIS) intelligence quotient $<90$ according to duration of breast-feeding. The differences between the groups were significant $(p<0.001)$. 
The majority of studies examining the effect of breast-feeding on cognitive development have found an advantage of breast-feeding, which remains when the results are controlled for potential confounders. This was also the case in the few studies that were able to control for maternal IQ. Positive effects in young adulthood were found in two non-overlapping samples using two different IQ tests. This finding is in accordance with results from the metaanalysis by Anderson et al. (11), where there was no difference between the effects measured during the first years of life and the effects seen later in childhood or around puberty. Thus, it seems as though the effects of breast-feeding on cognitive development, which are seen during the first years of life, are stable throughout childhood and into young adulthood.

There are several possible explanations for the positive effects of breast-feeding observed in these studies. A plausible explanation is the differences in composition between human milk and infant formula, e.g. differences in $n-3$ fatty acids, especially DHA. LCPUFAs, especially DHA, are major components of cell membranes in the central nervous system and retina. Several lines of evidence suggest that cellular functions are affected by the lipid composition of cell membranes (1). The content of $n-3$ in modern infant formula is high compared with the content in cow's milk and infant formula used in the early 1960s. Thus, the effects of breast-feeding would be expected to be somewhat larger in a cohort born in the early 1960s, compared with a cohort receiving modern infant formula, if $n-3$ fatty acid intake is the cause.

Factors associated with the feeding situation, i.e. physical or psychological contact between mother and child, could also have an effect. If this is the case, these factors are also a direct effect of breastfeeding. Another possibility is that mothers who choose to breast-feed on average stimulate and support their infants better than mothers who formula-feed. Unidentified factors that correlate with both infant feeding and the development of cognitive and intellectual ability (i.e. residual confounding) may also be involved. The mother's breast-feeding duration reflects, to some degree, the mother's concern with her infant's welfare, and her motivation and ability to stimulate and enrich her child's subsequent development, which are partly correlated with social class and education (14).

\section{References}

1. Lauritzen L, Hansen HS, Jørgensen MH, Michaelsen KF. The essentiality of long chain $n-3$ fatty acids in relation to development and function of the brain and retina. Prog Lipid Res 2001; 40: 1-94.

2. Jacobson JL, Jacobson SW. Intellectual impairment in children exposed to polychlorinated biphenyls in utero. N Engl J Med 1996; 335: 783-9.

3. Grandjean P, Weihe P, White RF, et al. Cognitive deficit in 7-year-old children with prenatal exposure to methylmercury. Neurotoxicol Teratol 1997; 19: 417-28.

4. Jacobson JL, Jacobson SW. Postnatal exposure to PCBs and childhood development. Lancet 2001; 358: 1568-9.

5. Walkowiak J, Wiener JA, Fastabend A, et al. Environmental exposure to polychlorinated biphenyls and quality of the home environment: effects on psychodevelopment in early childhood. Lancet 2001; 358: $1602-$ 7.

6. Skoczenski AM, Norcia AM. Development of VEP Vernier acuity and grating acuity in human infants. Invest Ophthalmol Visual Sci 1999; 40: 2411-7.

7. Jørgensen $\mathrm{MH}$, Hernell $\mathrm{O}$, Lund $\mathrm{P}$, et al. Visual acuity and erythrocyte docosahexaenoic acid status in breastfed and formula-fed term infants during the first four months of life. Lipids 1996; 31: 99-105.

8. Jørgensen MH, Hølmer G, Lund P, et al. Effect of formula supplemented with docosahexaenoic acid and gamma-linolenic acid on fatty acid status and visual acuity in term infants. J Pediatr Gastroenterol Nutr 1998; 26: 412-21.

9. Jørgensen MH, Hernell O, Hughes E, Michaelsen KF. Is there a relation between docosahexaenoic acid concentration in mothers' milk and visual development in term infants? J Pediatr Gastroenterol Nutr 2001; 32: 293-6.

10. SanGiovanni JP, Berkey CS, Dwyer JT, Colditz GA. Dietary essential fatty acids, long-chain polyunsaturated fatty acids, and visual resolution acuity in healthy fullterm infants: a systematic review. Early Hum Dev 2000; 57: 165-88.

11. Anderson JW, Johnstone BM, Remley DT. Breastfeeding and cognitive development: a meta-analysis. Am J Clin Nutr 1999; 70: 525-35.

12. Drane DL, Logemann JA. A critical evaluation of the evidence of the association between type of infant feeding and cognitive development. Paediatr Perinat Epidemiol 2000; 14: 349-56.

13. Mortensen EL, Michaelsen KF, Sanders SA, Reinisch JM. The association between duration of breastfeeding and adult intelligence. JAMA 2002; 287: 2365-71.

14. Jacobson SW, Jacobson JL. Breastfeeding and IQ: evaluation of the socio-environmental confounders. Acta Paediatr 2002; 91: 258-66.

Kim Fleischer Michaelsen

Department of Human Nutrition, The Royal Veterinary and Agricultural University, Frederiksberg, Denmark

E-mail: kfm@kvl.dk 